\title{
In-Medium Modifications of Hadron Properties
}

\author{
A. Tawfik* \\ Hiroshima University, 1-7-1 Kagami-yama, Higashi-Hiroshima Japan
}

\begin{abstract}
The in-medium modifications of hadron properties are briefly discussed. We restrict the discussion to the lattice QCD calculations for the hadron masses, screening masses, decay constants and wave functions. We review the progress made so far and describe how to broaden its horizon.
\end{abstract}

\section{Introduction}

"The origin of the mass" is one of the fundamental questions in physics. The mass of one composite system, like nucleus, is given by the summation of the masses of its constituents plus the binding energy. The proton and neutrons are composite systems too. But their masses can't be obtained according to this principle. We have to assume that the mass is mainly given by the kinetic energy of the quark and gluon fields. This energy has to be smaller than the potential energy which confines the qaurks in the hadron bound state. It is likely that the potential energy can be modified in thermal and dense medium. This explanation for the origin of the mass is likely valid in the chiral limit. As soon as the quarks get bare masses, the chiral symmetry will be spontaneously broken. Consequently, a mass difference appears between hadron states with same spin but different parity quantum numbers. This part of the mass will disappear, when the breaking of the chiral symmetry is repealed at a critical temperature $T_{c}$. According to the lattice QCD, the chiral symmetry breaking might partly be restored below $T_{c}$. Therefore, the hadron masses are conjectured to be modified at finite temperatures and densities.

Almost all hadron properties are extracted from the exponential decay of the two-point correlation functions. In the lattice QCD, the quark fields are discritized on the lattice sites. Any resulting operator has to conserve the lattice symmetry. Such an operator can be projected into the different hadron states. In present work, we restrict the discussion to the lattice QCD calculations. We review the progress made so far. We discuss how to extend this powerful tool to finite temperatures and densities.

Understanding the modifications of the hadron properties at finite temperatures and densities is very essential to study the behavior of the "hadronic" matter under extreme conditions. It is also important in order to interpret the phenomenology of hadron production in heavy-ion collisions. It would not be possible to give a solid description for the dynamics of the phase transitions, when these modifications are not taken into account [1].

The most extreme(st) modification occurs along the phase boundary. Not only a rapid change in the degrees of freedoms is most likely expected, but also a radical rearrangement of the correlation and entropy.

\section{Hadron mass spectrum on the lattice}

We aim to investigate the "origin of the mass" from first principle. As mentioned above, all hadron properties including the mass can be extracted from the two-point correlation

*tawfik@physik.uni-bielefeld.de 


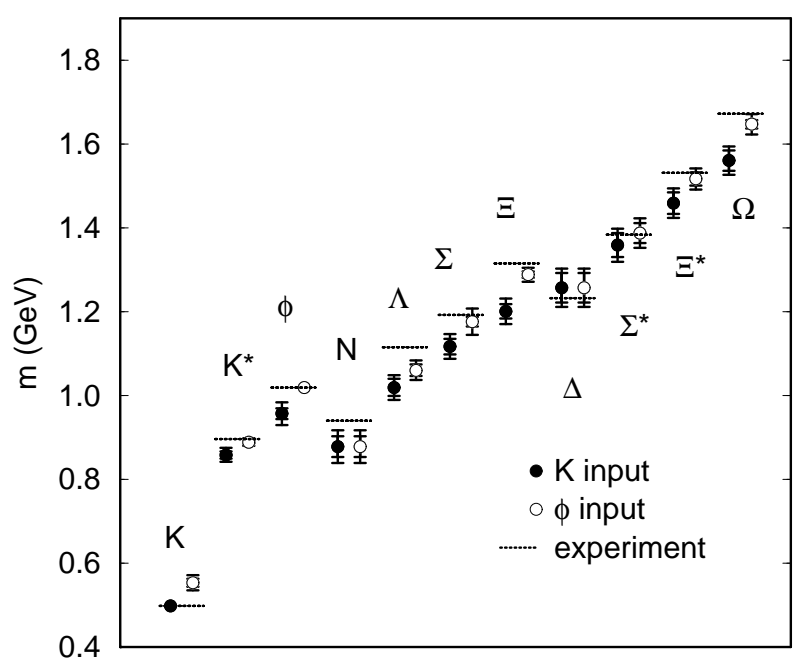

Fig. 1: Comparison between lattice results on light hadron spectrum at zero temperature and vanishing density and the experimental values [2].

functions. The generic meson operators are given as:

$$
\mathcal{O}_{m}(x)=\bar{\psi}_{\alpha}^{a}(x) \Gamma_{\alpha \beta} \psi_{\beta}^{b}(x)
$$

where $\Gamma_{\alpha \beta}$ are the Dirac matrices $\left(1, \gamma_{\mu}, \gamma_{5}, \gamma_{\mu} \gamma_{5}, \sigma_{\mu \nu}\right)$. The subscripts give the color quantum numbers. For the baryons

$$
\mathcal{O}_{b}(x)=\epsilon_{i j k}\left(\psi_{\alpha}^{i, a}(x) \Gamma_{\alpha \beta} \psi_{\beta}^{j, b}(x)\right) \psi_{\gamma}^{k, c}
$$

Then we can write down an expression for the two-point correlation function and its exponential decay in the Euclidean time. For pions, the operator needed to generate the spectrum reads

$$
P^{\dagger}(\vec{p}, \tau)=\sum_{\vec{x}_{n}} e^{i \vec{p} \cdot \vec{x}_{n}} \bar{u}^{a}\left(\vec{x}_{n}, \tau\right) \gamma_{5} d^{a}\left(\vec{x}_{n}, \tau\right)
$$

And at time $\tau$ the propagator is

$$
C(\vec{p}, \tau) \equiv<P(\vec{p}, \tau) P^{\dagger}(\vec{p}, 0)>=\sum_{h} \frac{A_{h}(\vec{p})}{2 E_{h}(\vec{p})} \exp \left(-E_{h}(\vec{p}) \tau\right)
$$

where $A_{h}(\vec{p})$ represent of overlap of the operator from hadron state $\mid h>$ with momentum $\vec{p}$. Below, we will show that the decay constant is hidden in this parameter. Then the propagator can be integrated over the Euclidean time- and two spatial-directions. The mass will be extracted from $E(\vec{p}) . E_{h}^{2}(\vec{p})=\vec{p}^{2}+m_{h}^{2}$ is the energy of hadron state. An example for the hadron mass spectrum at zero momentum is given in Fig. 1.

To extend these calculations over the region of finite temperatures, we need temporal two-point correlation functions.

$$
\begin{aligned}
<\mathcal{O}_{m}(x)>= & \frac{1}{\mathcal{Z}} \int d \psi d \bar{\psi} d U\left(\bar{\psi}^{a}(x) \Gamma_{\alpha \beta} \psi^{a}(x)\right)\left(\bar{\psi}^{a}(0) \Gamma_{\alpha \beta} \psi^{a}(0)\right)^{\dagger} \\
& \exp \left(-\bar{\psi} \mathcal{M} \psi-S_{G}\right) \\
<\mathcal{O}_{m}(x, \tau)>= & \frac{1}{\mathcal{Z}} \int d U \Gamma_{\alpha \beta} \mathcal{M}^{-1}(x, 0) \Gamma_{\alpha \beta}^{\dagger} \mathcal{M}^{-1}(0, x) \exp \left(-S_{G}\right)
\end{aligned}
$$



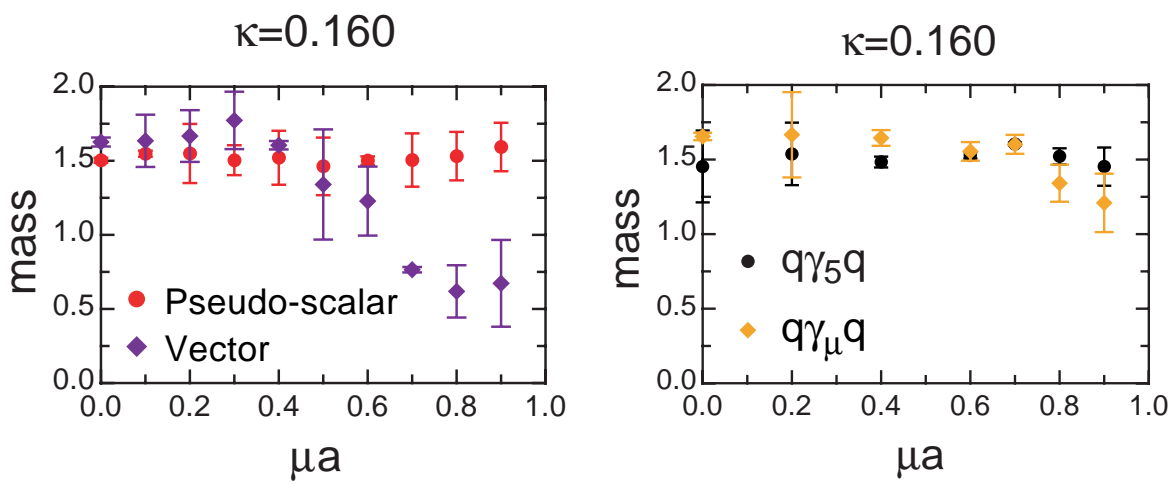

Fig. 2: Left panel: meson masses as function of chemical potential $\mu a$. The mass of pseudoscalar meson does not change with $\mu a$. The mass of vector meson drops with increasing $\mu a$. Right panel: the masses of baryon-type states are depicted in dependence on $\mu a$. No special behavior can be observed here.

where $\mathcal{M}=\gamma_{\mu} D_{\mu}-m_{q}$ is the fermion determinant and $S_{G}$ is the gauge action. $U$ stands for the link variable on the lattice.

The lattice calculations at finite chemical potential $\mu_{q}$ are not trivial, since the fermion determinant becomes complex and therefore the MC simulations are no longer applicable. Introducing imaginary chemical potential can solve this problem. We can also apply the Taylor series of the operator of interest at zero $\mu_{q}$.

$$
\mathcal{O}\left(\mu_{q}\right)=\left.C\right|_{\mu_{q}=0}+\left.\left.\mu_{q} \frac{d C}{d \mu_{q}}\right|_{\mu_{q}=0} \frac{\mu_{q}^{2}}{2} \frac{d^{2} C}{d \mu_{q}^{2}}\right|_{\mu_{q}=0}+\cdots
$$

The first estimation for the hadron masses at finite chemical potential $\mu$ is reported in Ref. 3]. The numerical study of two-color QCD with Wilson fermions on $4^{3} \times 8$ lattice shows that the mass of the pseudoscalar meson is almost constant at finite $\mu$. The vector meson shows a different behavior. The mass of rho-meson decreases with increasing $\mu$. This behavior qualitatively agrees with the predictions from the effective models, section 6 , In the baryonic channel, there is no change observed. It is important to note that these results must be taken as a qualitative indication about the behavior of the masses at finite chemical potential. As it was not possible to define the physical units, both mass and $\mu$ are given in units of lattice spacing $a$.

\section{The Debye screening mass}

In contrast to the temporal correlation functions from which we construct the hadron masses and decay constants, the spatial correlation functions are often analyzed on the lattice. The reason is the limited time direction on lattice. The screening masses can be constructed from the spatial correlation functions. At low temperatures, the screening masses are identical to the pole masses. At high temperatures, they are different. At very high temperatures, the screening masses are expected to approach $n \pi T . n \pi T$ gives the lowest Matsubara frequency.

The screening mass measures different quantities. It depends on the properties of the many-body system. Therefore, it is a useful tool to invistigate the plasma phase. The response of the medium to the existence of a weak perturbation, like hadron, appears in form of dynamical Debye screening of the long-range Coulomb potential. There are different definitions for the screening mass. The most common one relates it to the inverse Debye screening length. Also it is defined as the static limit of the longitudinal vacuum polarization tensor $\prod_{00}(p \rightarrow 0)=M_{D}$. 


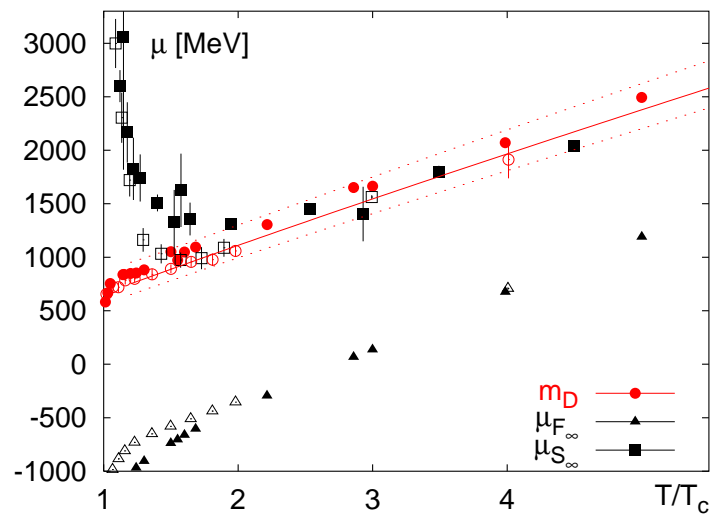

Fig. 3: The Debye screening mass, $m_{D}(T)$ for 2-flavor (open circles) and quenched QCD (solid circles) as function of $T / T_{c}$ [4]. Different mass scales are also included.

The screening mass on lattice can be obtained from the exponential falloff of a correlator of two Polyakov loops in the spatial extension. The latter is related to the free energy in the limit $x \rightarrow \infty$.

$$
\Delta F(x, T) \propto \frac{\alpha_{s}(T)}{x} \exp \left[-M_{D}(T) x\right]
$$

where $\alpha_{s}(T)=g^{2}(T) / 4 \pi$ is the strong coupling. Because of the interaction with the medium via gluon exchange, the gluon acquires an additional mass, called chromoelectric mass $m C E$. $m C E$ results from the finite infrared limit of the polarization tensor. It is proportional to $g(T) T$ in leading order perturbation theory.

As mentioned above, Eq. 4 can be extended to finite temperature by adding temperature-depending terms. The integral of the resulting correlation function over the Euclidean time- and two spatial-dimensions results in the screening mass.

$$
E_{h}^{2}(\vec{p}, T)=m_{H}^{2}+\vec{p}^{2}+\prod(\vec{p}, T)
$$

Fig. 3 depicts the results on the screening mass for quenched and 2-flavor QCD at zero chemical potential. The free energy given in Eq. 8, can be separated into internal energy and entropy. One finds that the internal energy of this quark-antiquark system is related to the screening mass. The dependence of screening mass on the temperature $M_{D}(T)$ measures the strength of the screening response of the medium due to inserting hadron in it. Different mass scales are also included. We find that the effects of the hadron mass on the screening response are much weaker than the effects of the energy and coupling.

In the perturbation theory, the screening mass - next-to-leading order - reads

$$
\begin{aligned}
m_{D}(T) & =m_{D}^{(L O)}+\frac{N g(T) T}{4 \pi} \ln \left(\frac{m_{D}^{(L O)}}{g^{2}(T) T}\right)+c_{N} g^{2}(T) T+\mathcal{O}\left(g^{3}(T) T\right) \\
m_{D}^{(L O)} & =\left(\frac{N_{c}}{3}+\frac{N_{f}}{6}\right)^{1 / 2} g(T) T
\end{aligned}
$$

where $c_{N}$ is a non-perturbative parameter. Therefore, it can be determined by nonperturbative methods, i.e. lattice.

Including the chemical potential is a non-trivial task [5]. In leading order approximation, the screening mass at $\mu_{q} \neq 0$ takes the form

$$
m_{D}\left(T, \mu_{q}\right)=\left(N_{c} / 3+N_{f} / 6+\left(\mu_{q} / T\right)^{2} N_{f} / 2 \pi^{2}\right)^{1 / 2} g(T) T
$$


Alternatively, we can use the non-perturbative methods to calculate $m_{D}\left(T, \mu_{q}\right)$. We can use the response of the screening mass at $\mu_{q}=0$ [6]. As in Eq. 17 we can apply Taylor expansion at fixed temperature, coupling and bare quark mass to calculate $m_{D}\left(T, \mu_{q}\right)$

$$
\frac{m_{D}\left(T, \mu_{q}\right)}{T}=\left.\frac{m_{D}(T)}{T}\right|_{\mu_{q}=0}+\left.\left(\frac{\mu_{q}}{T}\right) \frac{\partial m_{D}(T)}{\partial \mu_{q}}\right|_{\mu_{q}=0}+\left.\left(\frac{\mu_{q}}{T}\right)^{2} \frac{T}{2} \frac{\partial^{2} m_{D}(T)}{\partial \mu_{q}^{2}}\right|_{\mu_{q}=0}+\cdot
$$

The coefficients are determined by measuring the hadron propagators and their derivatives at $\mu=0$ by using the standard MC method.

$$
\begin{aligned}
C_{\pi}(z) & =C_{1}\left(e^{-\hat{m}_{1} \hat{z}}+e^{-\hat{m}_{1}\left(N_{z}-\hat{z}\right)}\right)+C_{2}\left(e^{-\hat{m}_{2} \hat{z}}+e^{-\hat{m}_{2}\left(N_{z}-\hat{z}\right)}\right) \\
C_{\rho}(z) & =C_{1}^{\prime}\left(e^{-\hat{m}_{1}^{\prime} \hat{z}}+e^{-\hat{m}_{1}^{\prime}\left(N_{z}-\hat{z}\right)}\right)+C_{2}^{\prime}(-1)^{z}\left(e^{-\hat{m}_{2}^{\prime} \hat{z}}+e^{-\hat{m}_{2}^{\prime}\left(N_{z}-\hat{z}\right)}\right) \\
C_{N}(z) & =C_{1}^{\prime \prime}\left(e^{-\hat{m}_{1}^{\prime \prime} \hat{z}}+(-1)^{z} e^{-\hat{m}_{1}^{\prime \prime}\left(N_{z}-\hat{z}\right)}\right)+C_{2}^{\prime \prime}\left((-1)^{z} e^{-\hat{m}_{2}^{\prime \prime} \hat{z}}+e^{-\hat{m}_{2}^{\prime \prime}\left(N_{z}-\hat{z}\right)}\right)
\end{aligned}
$$

$N_{z}$ is the length of the lattice in the $z$-direction. The results for degenerate quark masses $a m_{q}=0.05$ are summarized in Fig. 4. We note that the screening mass in all hadron channels increases with increasing the temperature. This means that the response of the heat path to the existence of the hadrons is enhanced as the temperature raises. Above $T_{c}$ this behavior seems to be stronger.

The influence of chemical potential starts to set on above $T_{c}$. Below $T_{c}$, there is almost to influence. In the deconfined phase the screening mass at finite $\mu_{q}$ is larger than its value at $\mu_{q}=0$. The mesons are much sensitive than the baryons. It is important to note that we get different screening masses depending on the hadron state. This means that the heat bath (gluonic system) is polarized around the static charge (the hadron) and its parton density seems to be modified, accordingly.

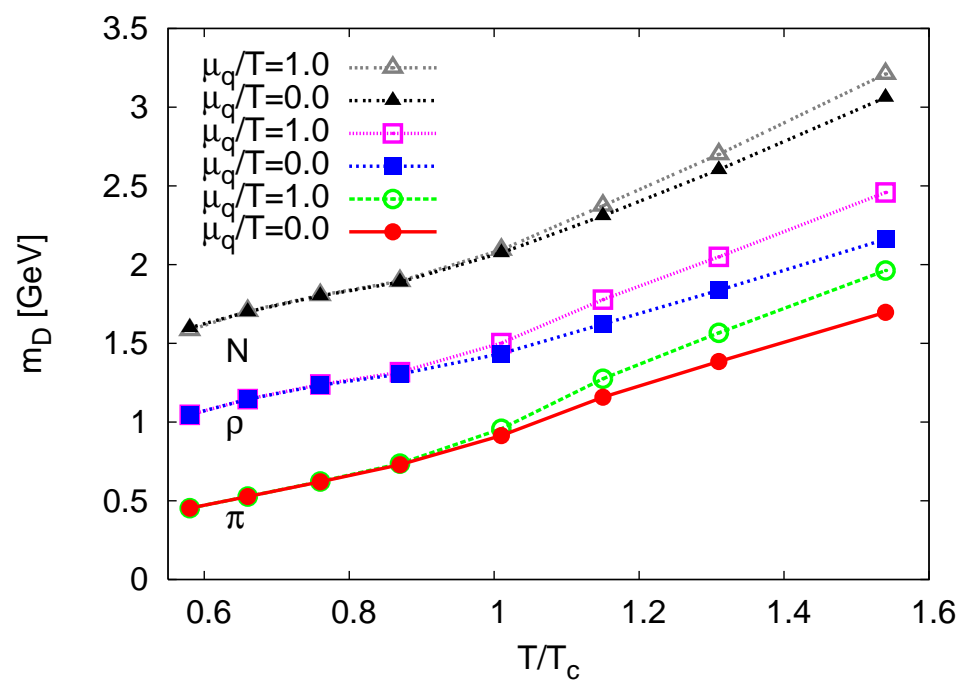

Fig. 4: The screening mass, $m_{D}$ as function of $T / T_{c}$. Three hadron states are shown here. For each hadron we depict the results at $\mu_{q} / T=0.0$ and $\mu_{q} / T=1.0$. We set the physical units according to $T_{c}=0.225 \mathrm{GeV}$.

\section{The Decay Constant}

In the continuum theory, the pion decay constant is defined as

$$
\sqrt{2} f_{\pi} m_{\pi}=\left\langle 0\left|\bar{u} \gamma_{4} \gamma_{5} d\right| \pi^{+}(\vec{p}=0)\right\rangle
$$


We can use the temporal correlation functions given in Eq. 6 to calculate the decay constant for the hadron of interest. At large separations and low temperatures the pole contributions dominate the correlation function.

$$
<\mathcal{O}(\vec{p}, \tau)>=\frac{\mathcal{C}(\vec{p})}{2 E_{h}(\vec{p})} \frac{\cosh \left(E_{h}(\vec{p})\left(\tau-\tau_{0}\right)\right)}{\sinh \left(E_{h}(\vec{p}) \tau_{0}\right)}
$$

where $\mathcal{C}(\vec{p})$ is the residue of the current when $\vec{p} \rightarrow 0$. Applying the GMOR relation, we can construct the pseudoscalar correlation function, for instance,

$$
<\mathcal{O}(\tau)>_{\pi}=f_{\pi}^{2} \frac{m_{\pi}^{2}}{8 m_{q}^{2}} \frac{\cosh \left(m_{\pi}\left(\tau-\tau_{0}\right)\right)}{\sinh \left(m_{\pi} \tau_{0}\right)}
$$

There are extensive lattice calculations for the decay constants of different hadrons at zero temperature [7. This quantity is essential for the construction of CKM (CabibboKabayashi-Maskawa) unitary triangle (quark mixing matrix). Its shape is directly related to the properties of the weak interactions and the phenomenon of CP-violation.

Including chemical potential requires two types of modifications. The first one deals with the influence of the finite density of the hadron mass. This has been discussed above. The second one deals with the influence on the decay constant itself.

$$
\left(f_{\pi}^{*}\right)^{2}\left(m_{\pi}^{*}\right)^{2}=-2 m_{q}<\bar{q} q>^{*}
$$

The superscripts $*$ denote the in-medium modifications. $\langle\bar{q} q\rangle^{*}$ is the quark condensates at finite temperature and density. In Fig. [5 we show the results from the resonance gas model [8]. At least according to the quark condensates, we can easily expect that the decay constant has to be modified in the medium. To this end, we still need to study the in-medium modification of the hadron masses.
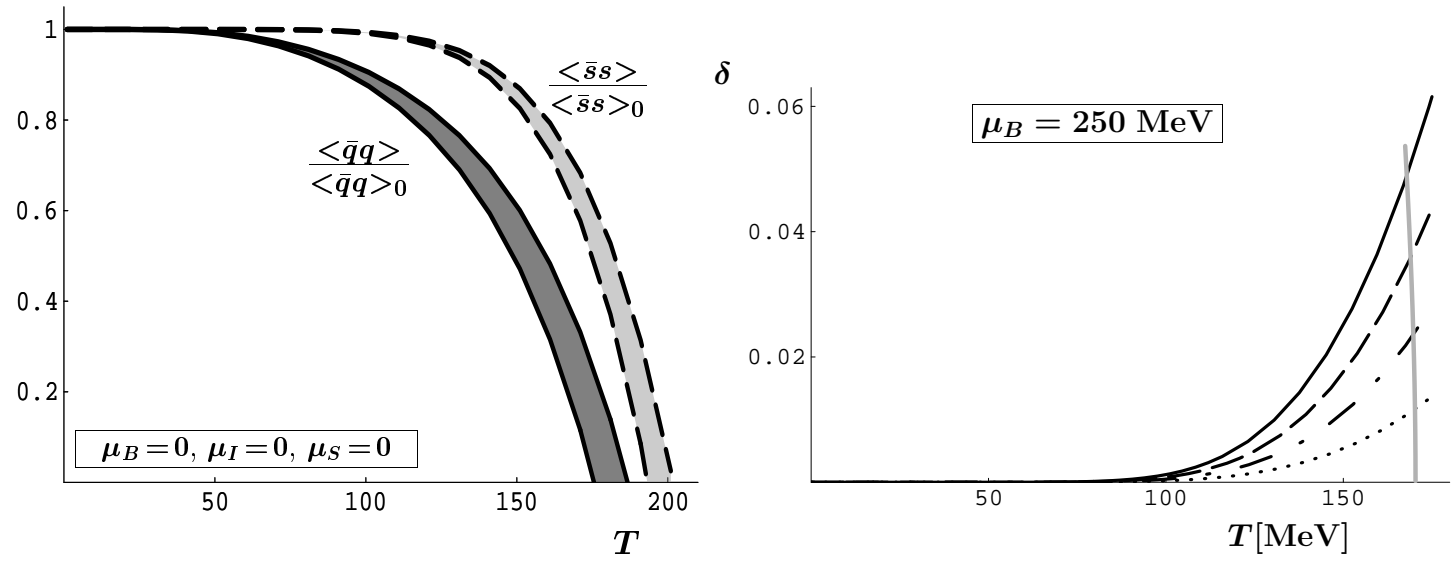

Fig. 5: Left panel: light and strange quark condensate as function of temperature. Right panel: the difference between light quark condensates normalized to the vacuum value calculated at a fixed baryochemical potential but different isospin chemical potentials. Depending on the medium, the quark condensates vanish at different critical temperatures.

\section{Wave function}

We assumed that the hadronic state given in Eqs. 10 2 have to fulfill the lattice symmetry. But they still need a spatial dimension. The quarks have to be positioned at a certian distance apart from each other. Even in the confined phase, the quarks are not allowed to 
shear one location, since they are fermions. Numerically, this requirement is convenient. We put the quarks at different lattice sites separated by a distance $r$, for instance. The separated quarks still have to remain connected to each other. To achieve this, we have to add gluon links. The resulting signals for the ground state have to be dominant in the contrast to that for the excited states $[9$. For example, the pion state can be expressed as

$$
\left|\pi>=\alpha_{1}\right| q \bar{q}>+\alpha_{2}\left|q \bar{q} g>+\alpha_{3}\right| q \bar{q} g g>+\cdots
$$

This is equivalent to adding smeared gauge fields. The added gauge fields will build up a gluonic cloud around the quark-antiquark system. What would this mean, technically? It means that one plaquette has to be rescaled. And in the corresponding link variable a distance $r$ will appear, $\psi(\vec{x}, r)$. Consequently, the operators, Eqs. 11 2, have to be modified. From this smearing procedure, the correlation functions becomes oscillating in $r$. The oscillation amplitude defines the wave function of the corresponding hadron state.

The bound states can be studied by means of Bethe-Salpeter (BS) function. The norm of the BS amplitude $|<0| \mathcal{O}(0)|h, \vec{p}>|$ is equivalent to the parameter $A_{h}^{1 / 2}(\vec{p})$ given in Eq. 4. The BS amplitude gives the probability of finding a pre-defined state. This new (smeared) operator has to remain gauge invariant. The gauge invariant BS amplitude for one pion with momentum $\vec{p}$ is

$$
A_{\pi}(\vec{p}, \vec{x})=<0\left|\bar{d}(\overrightarrow{0}) \gamma_{5} \mathcal{G}(\overrightarrow{0}, \vec{x}) u(\vec{x})\right| \pi(\vec{p})>
$$

where $\mathcal{G}(\overrightarrow{0}, \vec{p})$ gives the path-ordered product of the gauge links that connect the point $\overrightarrow{0}$ with $\vec{x}$. The normalized two-point correlators construct the amplitude

$$
A_{\pi}(\vec{p}, \vec{x} ; \tau)=\frac{<0\left|\bar{d}(\overrightarrow{0} ; \tau) \gamma_{5} \mathcal{G}(\overrightarrow{0}, \vec{x} ; \tau) u(\vec{x} ; \tau) \quad \bar{u}(\vec{y} ; 0) \gamma_{5} d(\vec{y} ; 0)\right| 0>}{<0\left|\bar{d}(\overrightarrow{0} ; \tau) \gamma_{5} u(\vec{x} ; \tau) \quad \bar{u}(\vec{y} ; 0) \gamma_{5} d(\vec{y} ; 0)\right| 0>}
$$

From this procedure, it is now obvious, that the wave function is also constructed from correlation function of two operators. The first one represents the hadron state. The second one gives the annihilation process. This picture is supported by the smearing process, as we described above. Implementing this procedure under gauge invariance assumption straightforwardly leads to calculating the wave function on lattice.

The wave function is an essential quantity to investigate the bound state. The influence of thermal and dense medium on the wave function would reflect to what extend can the bound state survive. On the other hand, one needs to elaborate the bound state potential in such a medium. We know from perturbative and non-perturbative calculations, that the heavy quark potential gives a solid description for the effective potential. It has been found that the effective potential of two quarks with a short separation is a Coulomb-type. When the quarks are pulled out to a very large distance, the effective potential becomes linear.

\section{Heavy-ion Collisions}

In this section, we discuss mass scaling laws which explain on how the strong coupling change in dense and thermal medium. Using effective Lagrangian which assumes spontaneously breaking of chiral symmetry, Brown and Rho suggested a mass scaling in medium [10, in order to explain the large mass enhancement of the lepton pairs observed in CERES experiment.

$$
\frac{m_{\rho}^{*}}{m_{\rho}} \approx \frac{f_{\pi}^{*}}{f_{\pi}} \sim\left(\frac{<\bar{q} q>^{*}}{<\bar{q} q>}\right)^{1 / 2}
$$


Subsequently, the reduction of the masses of baryons and mesons are equally scaled; $m_{B}^{*}=$ $x m_{B}$ and $m_{M}^{*}=x m_{M}$. The validity of this scaling law is restricted within the confined phase, especially in the region after the freeze out. The gradual restoration of the chiral symmetry breaking is essential to estimate this scaling law.

Based on QCD sum rules Hatsuda and Lee [11, suggested another scaling law.

$$
\frac{m_{\rho}^{*}}{m_{\rho}}=1-a \frac{n_{B}}{n_{0}}
$$

where $n_{B}$ is the baryon density and $n_{0}$ is the density of normal nuclear matter. The parameter $a$ has a value ranging between 0.15 and 0.18 .

From an effective theory with hidden local symmetry [12, it is expected that the mass of vector meson entirely vanishes at $n_{0}$. At this density, the pion decay constant will vanish too. Furthermore, a softening of the masses of $\sigma$ and $\rho$ mesons is expected to be associated with the gradual restoration of chiral symmetry breaking.

The results given in Fig. [6] illustrate one application of these models. The invariant mass and width modifications in heavy-ion collisions are shown. These recent results from different nuclear media obviously show an access. This access can only be explained, when the mass is scaled according to Eq. 21 with $a=0.15$. The first direct measurement of the decay channel $\rho^{0}(770) \rightarrow \pi^{+} \pi^{-}$(branching ratio 100\%) is reported in Ref. 13. Also a mass shift from $p+p$ to peripheral $A+A$ collisions has been observed. As a possible explanation for this modifications one suggests the dynamical interactions with the surrounding matter and the distortions of the correlations and phase space.
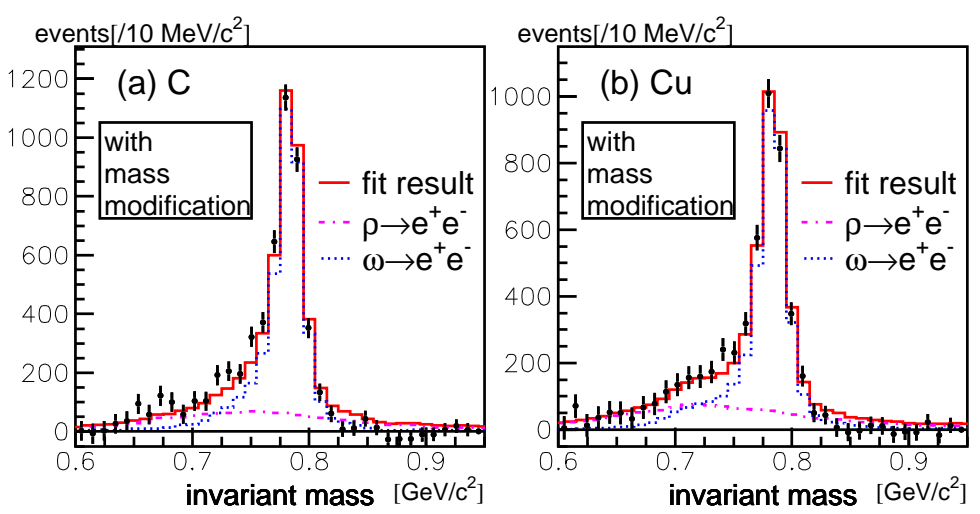

Fig. 6: The invariant mass spectrum in two different targets (media) is measured at KEK. The observed access can only be explained, when the modification of masses in the medium is taken into consideration [14]

Another significant in-medium modification has been observed while comparing the production and absorption in $p+p, p+A$ and $A+A$ collisions. Fig. 7 shows the strangeness production and its in-medium modification. We observe an obvious modification, when the medium is changed. Going from $p+p$ to $A+A$ the medium density is significantly increased. It is also interesting to note that the modification depends on the hadron of interest. For $K^{-}$, the modification is larger than for $K^{+}$. The reason is the different influences of the medium on the particle production and absorption. The production of $K^{-}$mainly depends on whether there are hyperons in the medium. The absorption of both kaons depends on the number of nucleons. 


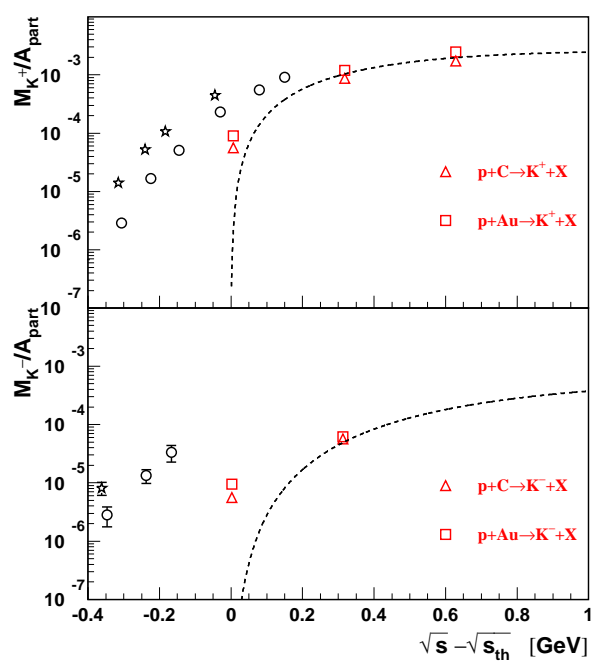

Fig. 7: Particle production of $K^{-}$(top panel) and $K^{+}$(bottom panel) as function of the excess energy [15]. The excess energy represents the difference between $\sqrt{s_{N N}}$ and the energy required for $K^{-}\left(K^{+}\right)$production.

\section{Summary}

We have discussed the in-medium modifications of the hadron properties. We restricted the discussion to the lattice QCD calculations for the hadron mass, Depye screening mass, decay constant and wave function. We have given a brief review of the progress made so far and discussed how to extend the lattice calculations to finite temperatures and densities.

There are precise lattice calculations for the masses of low-lying hadron states at zero temperature. Studying the thermal effects on the hadron mass is essential to understand the hadron production and absorption in heavy-ion collisions. Should the hadron mass fulfill the description given by Hagedorn in 1960's and by the dual models in the beginning of 1970's, our understanding of the phase diagram and the transition at high temperatures and densities has to be revised (modified). The dynamics controlling such a phase transition will be different, if the hadron resonances shall keep or lose their finite volumes (finite wave functions) and if their masses shall be modified or remain unchanged. We might need to mention here that the hadron resonance gas calculations [16] did not assume finite volume and mass scaling in dense and thermal medium.

The screening mass gives the response of the medium to the existence of a weak perturbation like the statically charged hadron. On the other hand, this quantity describes the properties of the medium. We get information on the parton density, entropy production and the long-range correlations in the medium. As we have seen, there are different lattice calculations using different approaches. We still need to study the medium response to the low-lying parity partners, like $\sigma$ for $\pi, a_{1}$ for $\rho$ and $N(1536)$ for $N$. Also we need to study the screening mass of deconfined quarks in thermal and dense plasma.

We have also discussed the decay constant. To the auther's knowledge there is no lattice calculations at finite temperature. At least there is no systematic study for the thermal effects. Using the decay constant, we can study the restoration of the chiral symmetry breaking. On the other hand, the quark condensates in vacuum [17] are obviously modified in the medium. Including chemical potential is not trivial, because the temporal correlation function is related to the decay constant and simultaneously to the hadron mass. Both quantities have to be expressed as function of chemical potential.

The wave function provides essential information on the bound state. There is a framework to proceed calculations for the wave function on lattice. We have mentioned 
that the wave function can be expressed by the Bethe-Salpeter amplitude. Studying the hadronic bound states at finite temperatures and densities elaborates the possible interactions with the medium and to what extend can the bound state survive.

Finally we have discussed some phenomenological examples on the modification of some hadron properties.

\section{Acknowledgment}

I would like to thank the Yukawa Institute for Theoretical Physics at the Kyoto University where I have introduced this work at the YITP workshop on "Hadrons ant Finite Density 2006" YITP-W-05-24, Feb. 20-22, 2006. This work has been financially supported by the Japanese Society for the Promotion of Science.

\section{References}

[1] A. Tawfik, Phys. Rev. D 71054502 (2005); hep-ph/0602094

[2] CP-PACS Collaboration (S. Aoki et al.), Phys. Rev. D 67034503 (2003)

[3] S. Muroya, A. Nakamura, C. Nonaka, Prog. Theor. Phys. Suppl. 149247 (2003)

[4] O. Kaczmarek, F. Zantow, hep-lat/0512031

[5] P. Arnold, L. G. Yaffe, Phys. Rev. D 527208 (1995)

[6] S. Choe et al., Nucl. Phys. A 698395 (2002); A. Nakamura, et al., Nucl. Phys. Proc. Suppl. 119493 (2003); I. Pushkina et al., Phys. Lett. B 609265 (2005)

[7] T. Chiu, et al., hep-lat/0509172

[8] A. Tawfik, D. Toublan, Phys. Lett. B 62348 (2005); D. Toublan, hep-ph/0511138

[9] R. Gupta, D. Daniel, J. Grandy, Phys. Rev. D 483330 (1993)

[10] G. Brown, M. Rho, Phys. Rev. Lett. 662720 (1991)

[11] T. Hatsuda, S. H. Lee, Phys. Rev. C 46 R34 (1992)

[12] M. Harada, Phys. Rept. 3811 (2003);

M. Harada, C. Sasaki, Phys. Rev. D 73036001 (2006)

[13] STAR Collaboration (J. Adams et al.) Phys. Rev. Lett. 92092301 (2004)

[14] M. Naruki et al., nucl-ex/0504016

[15] W. Scheinast, et al., nucl-ex/0512028

[16] F. Karsch, K. Redlich, A. Tawfik, J. Phys. G 30 S1271 (2004); Phys. Lett. B 57167 (2003); Eur. Phys. J. C 29549 (2003)

[17] D. Miller, A. Tawfik, Acta Phys. Polon. B 352165 (2004); Acta Phys. Polon. B 36801 (2005); J. Phys. G 30731 (2004); hep-ph/0309139. hep-ph/0308192, hep-ph/0312368 